\title{
Advanced Noncoherent Detection in Massive MIMO Systems via Digital Beamspace Preprocessing
}

\author{
Stephan Bucher *(D) and Christian Waldschmidt \\ Institute of Microwave Engineering, Ulm University, 89081 Ulm, Germany; christian.waldschmidt@uni-ulm.de \\ * Correspondence: stephan.bucher@uni-ulm.de
}

Received: 15 October 2020; Accepted: 18 November 2020; Published: 23 November 2020

\begin{abstract}
Noncoherent detection in massive multiple-input/multiple-output (MIMO) uplink systems provides a low-complexity alternative to its coherent counterpart. Requiring no actual channel knowledge but the per-user induced power at the base station, comparable performance as channel-estimation-based detection can be achieved when the users are located in the near-field of the base station. However, noncoherent detection fails in scenarios where users are in the far-field due to an insufficient capability to separate the users in terms of their spatially induced power. For this purpose, a dielectric lens or an analog beamforming structure can be employed, which are capable to focus the power of the incident waves to a smaller subset of the antennas at the base station. These so-called analog beamspace techniques have been demonstrated to enable again the noncoherent detection scheme. Analogous to a spatial Fourier transform, beamspace techniques can be also realized in the digital domain offering more flexibility. Its applicability to noncoherent detection is studied in this paper. It is shown numerically that by means of digital beamspace preprocessing, considerable performance gains can be achieved. Applied in dominant line-of-sight channels, a large number of users can be accommodated and the residual performance gap to coherent detection with perfect channel knowledge is minimal.
\end{abstract}

Keywords: massive MIMO; noncoherent detection; beamspace processing

\section{Introduction}

The massive multiple-input/multiple-output (MIMO) technology embodies one of the key elements in future wireless communication systems [1-6]. Equipped with a very large number of antennas at the base station (BS), massive MIMO systems offer the potential to meet the ever-increasing demand for high throughput and energy efficiency. To this end, sufficiently accurate channel state information (CSI) is essential, which is gathered via pilot signaling using predefined (orthogonal) pilot symbols. Since the number of channel coefficients to be estimated is huge in massive MIMO systems, CSI acquisition can become a challenging task and may suffer from pilot contamination due the re-use of pilot symbols in neighboring communication cells [7]. Moreover, outdated CSI or estimation inaccuracies have a severe impact on the system performance [8,9]. Additionally, the channel-training overhead reduces the overall time, where data can be transmitted resulting in a loss of spectral efficiency [10].

In order to overcome the need of channel estimation and pilot symbol allocation, noncoherent detection schemes can be applied in the uplink as proposed in [11,12]. Here, actual channel knowledge is not required to recover the individual transmitted data streams but the per-user induced power over the receive antennas of the BS, namely the user-specific power-space profile (PSP) of the users. As long as the users can be separated at the BS in terms of their spatially induced power, noncoherent detection performs comparably to its CSI-based (coherent) counterpart $[13,14]$. However, the spatial separation can only be obtained in scenarios where the users are located in the 
near-field of a physically-large BS [15-18]. Consequently an adaption is needed to handle far-field users and to keep the overall size of the BS in an acceptable range for a practical implementation. To this end, (analog) beamspace techniques can be employed focusing the energy of the incoming waves to a smaller subset of the BS antenna array depending on the angle of arrival. This process can also be interpreted as a spatial Fourier transform. Exploiting the angular sparsity of the propagation channel, where the uplink transmit signals of the users impinge at the BS from sufficiently different angular directions, the users can be separated enabling again the noncoherent detection scheme. This has been successively demonstrated for a lens-embedded and an analog beamforming-based system $[19,20]$. So far, lens antenna arrays were investigated solely in the context of coherent massive MIMO transceivers aiming at a reduction of hardware costs in both uplink and downlink [21-26]. The same applies to the studies in [27-31], where an alternative representation of the lens is used on the concept of beamspace MIMO.

To achieve the crucial user separation for noncoherent detection in far-field scenarios, the proposed analog beamspace techniques in $[19,20]$ require additional bulky hardware, which can suffer from non-idealities, high complexity, and lacking adaptability to specific channel conditions. Thus, an all digital implementation is of major interest avoiding the stated issues and providing the potential of even improved user separability via adapted signal processing at the same time.

This work studies beamspace techniques in the digital domain and its application to the noncoherent detection scheme. To this end, the relevant requirements are identified and detailed to conceive a highly advanced and competitive noncoherent detection scheme compared to the classical and initial approach proposed in $[11,12]$ by means of digital beamspace preprocessing. Originally, a high-quality phase reference and a perfect synchronization of the local oscillators (LOs) is not required in case of noncoherent detection. Consequently it can benefit from a low-complexity LO distribution network, where independent LOs for each receive branch can be employed for instance [17]. This applies also for the analog implementation of the beamspace techniques as in $[19,20]$. However, the application of the digital beamspace preprocessing requires a coherent demodulation of the receive branches to preserve the phase progression between adjacent receive channels. This can become a very challenging task, especially in massive MIMO systems, where the number of receive paths is quite large. Therefore, an extension to a sub-array architecture is explored in the context of digital beamspace preprocessing as well offering the opportunity to relax the design requirements of the LO distribution network. In summary, the contribution of this work is to combine the individual (known) concepts of noncoherent detection scheme, sub-array approach, and digital beamspace processing for a joint examination under proper channel conditions. To the authors' best knowledge, this is presented for the first time in the area of massive MIMO transmission.

This paper is organized as follows. The system model is introduced in Section 2, which includes a generalized system overview when digital beamspace preprocessing is applied. After a brief recapitulation of the underlying channel model and the noncoherent detection scheme denoting the most important aspects, a detailed description of the digital beamspace preprocessing tailored to noncoherent detection is presented. Numerical results are shown in Section 3, where full- and sub-array architectures and the influence of the channel properties are discussed. Finally, Section 4 concludes the paper.

Notations: Bold-faced capital letters and bold-faced small letters denote matrices and column vectors, respectively. Superscripts $(\cdot)^{\mathrm{T}},(\cdot)^{\mathrm{H}}$, and $(\cdot)^{-1}$ indicate the transpose, the conjugate transpose, and the inverse of a matrix, respectively. The operators $\mathrm{E}\{\cdot\},\|\cdot\|_{2}$, and $\|\cdot\|_{\mathrm{F}}$ denote the expectation, the Euclidean norm of a vector, and the Frobenius norm of a matrix, respectively. $\boldsymbol{I}_{N}$ is the $N \times N$ identity matrix and diag $(\cdot)$ represents a diagonal (block) matrix. 


\section{System Model}

\subsection{System Overview}

In this paper, a multi-user uplink system is considered as depicted in Figure 1a. Here, $N_{\mathrm{u}}$ single-antenna users simultaneously transmit to a central BS, where a uniform linear array is employed with a very large number of receive antennas $N_{\mathrm{rx}} \gg N_{\mathrm{u}}$ and an antenna distance of $d_{\mathrm{a}}$. The users are assumed to be located in the far-field, i.e., the (radial) user distance $d_{u}$ meets the far-field condition with respect to the physical antenna array size $D=N_{\mathrm{rx}} d_{\mathrm{a}}$ at the BS and the wavelength $\lambda$ according to $d_{u}>2 D^{2} / \lambda$ [32]. Consequently, plane waves impinge at the receiver from various angular directions $\phi$ (measured with respect to the normal of the array axis) depending on the user positioning and characteristics of the propagation channel. In order to recover the individual data streams transmitted by the users, noncoherent detection schemes are applied at the BS. This requires the per-user received power induced at the antenna array of the BS to be spatially separated, which is not the case due to the far-field assumption. To this end, beamspace preprocessing is utilized in the digital domain after coherent downconversion and analog-to-digital conversion. Digitally and simultaneously generated beams pointing into different angular directions within a predefined field of view (FoV) focus the energy of the incoming waves to a smaller subset of the overall receive paths (see Figure 1a).

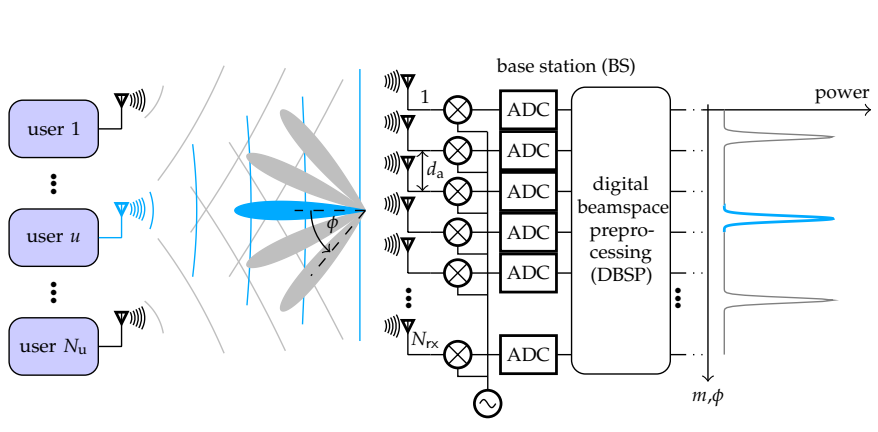

(a)

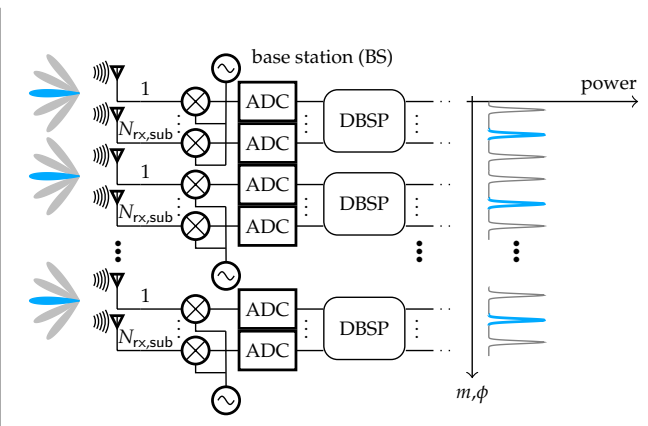

(b)

Figure 1. Digital beamspace preprocessing in noncoherent massive multiple-input/multiple-output (MIMO) systems: (a) Full-array and (b) sub-array architecture.

In the following, a joint digital beamspace preprocessing of all $N_{r x}$ receive branches is denoted as the full-array architecture. This system can exploit its full potential in terms of angular resolution and power-focusing capability. However, to form beams into a desired direction, a fully coherent demodulation of the received branches is required. The complexity of such a high-quality LO distribution network scales with the number of receive branches. In order to relax the design requirements, the full-array architecture can be divided into $N_{\text {sub }}$ sub-arrays of equal size (see Figure $1 \mathrm{~b}$ ), where coherency of the receive channels can be provided more easily and where one LO with proper distribution network can be employed instead. Consisting of $N_{\mathrm{rx}, \text { sub }}=N_{\mathrm{rx}} / N_{\text {sub }}$ antennas or rather receive branches, each sub-array can perform digital beamspace preprocessing individually, i.e., forming beams in the digital domain within a predefined FoV. Noteworthy, due to the reduced aperture size $D^{\prime}=D / N_{\text {sub }}$, the (radial) user distance could be less to meet the far-field condition in the sub-array case.

Further details regarding digital beamspace preprocessing can be found in Section 2.4. First, the underlying channel model and the noncoherent detection scheme are briefly reviewed.

\subsection{Channel Model}

The massive MIMO propagation channel is modeled using a geometry-based stochastic approach, more precisely using an adapted version of the COST 2100 channel model $[33,34]$. Here, 
randomly placed clusters in the topological simulation environment emulate physical scattering objects, where each cluster consists of groups of multi-path components (MPC). Its parameters, such as complex amplitude and angular direction, are computed on the basis of the simulation area. Generally, the clusters can be assigned to the following four categories: (i) local clusters (scatterers surrounding the users and/or the BS), (ii) single clusters (scattering on one object), (iii) twin clusters (scattering on multiple objects), and (iv) common clusters (propagation through the same set of clusters). In addition to multi-path propagation, a line-of-sight (LOS) between the users and the BS can be considered as well. In order to reduce the complexity of the channel and to ease the interpretation of the subsequent numerical results, the propagation via LOS and local clusters around the users are taken into account.

Considering omni-directional single-antenna users located in the far-field and a BS employing a uniform linear array of directional antennas with pattern $C_{\mathrm{rx}}(\phi)$, the complex channel vector $\boldsymbol{h}_{u}=\left[h_{1, u}, \ldots, h_{N_{\mathrm{r} x}, u}\right]^{\top}$ of the $u$-th user in case of a flat-fading channel is given by

$$
\begin{aligned}
\boldsymbol{h}_{u}= & C_{\mathrm{rx}}\left(\phi_{u}^{\mathrm{LOS}}\right) a_{u}^{\mathrm{LOS}} \boldsymbol{a}_{N_{\mathrm{rx}}}\left(\phi_{u}^{\mathrm{LOS}}\right) \\
& +\sum_{p=1}^{N_{p}} C_{\mathrm{rx}}\left(\phi_{u, p}^{\mathrm{MPC}}\right) a_{u, p}^{\mathrm{MPC}} \boldsymbol{a}_{N_{\mathrm{rx}}}\left(\phi_{u, p}^{\mathrm{MPC}}\right),
\end{aligned}
$$

where $a_{u}^{\mathrm{LOS}}$ is the complex amplitude of the LOS component and $\phi_{u}^{\mathrm{LOS}}$ its corresponding direction of arrival (DOA). The $p$-th MPC originating from $N_{p}$ local clusters is denoted by $a_{u, p}^{\mathrm{MPC}}$ with DOA $\phi_{u, p}^{\mathrm{MPC}}$. $\boldsymbol{a}_{N_{\mathrm{rx}}}(\phi)$ represents the steering vector of a uniform linear array with $N_{r x}$ elements of distance $d_{\mathrm{a}}$, i.e.,

$$
\boldsymbol{a}_{N_{\mathrm{rx}}}(\phi)=\frac{1}{\sqrt{N_{\mathrm{rx}}}}\left[\begin{array}{c}
1 \\
\mathrm{e}^{-\mathrm{j} k d_{\mathrm{a}} \sin (\phi)} \\
\vdots \\
\mathrm{e}^{-\mathrm{j} k\left(N_{\mathrm{rx}}-1\right) d_{\mathrm{a}} \sin (\phi)}
\end{array}\right],
$$

where $\phi$ denotes the DOA, $k=2 \pi / \lambda$ is the wavenumber, and $\lambda$ is the wavelength.

Since the local clusters are uniformly distributed in the azimuthal plane around the users, $a_{u, p}^{\mathrm{MPC}}$ and its DOA $\phi_{u, p}^{\mathrm{MPC}}$ becomes random for different channel realizations, where $\phi_{u, p}^{\mathrm{MPC}}$ can be generally characterized by an angular spread with standard deviation $\sigma_{\mathrm{s}}$. On the contrary, $\phi_{u}^{\mathrm{LOS}}$ is deterministic and the power of the LOS component is adjusted by a LOS-to-MPC ratio (LMR), which is defined as

$$
\mathrm{LMR}=10 \log _{10} \frac{\left|a_{u}^{\mathrm{LOS}}\right|^{2}}{\sum_{p=1}^{N_{p}}\left|a_{u, p}^{\mathrm{MPC}}\right|^{2}} \mathrm{~dB} .
$$

\subsection{Noncoherent Detection}

The received signal $r_{k}$ at the $N_{r x}$ BS antennas at time instant $k$ can be written as

$$
\boldsymbol{r}_{k}=\boldsymbol{H} \boldsymbol{b}_{k}+\boldsymbol{n}_{k}
$$

where $\boldsymbol{H}=\left[\boldsymbol{h}_{1}, \ldots, \boldsymbol{h}_{N_{\mathrm{u}}}\right]$ is the $N_{\mathrm{rx}} \times N_{\mathrm{u}}$ channel matrix collecting the complex-valued channel vectors $\boldsymbol{h}_{u}$ of the users as defined in (1), $\boldsymbol{b}_{k}=\left[b_{k, 1}, \ldots, b_{k, N_{u}}\right]^{\top}$ comprises the transmit symbols of each user $u$, and $n_{k}=\left[n_{1, k}, \ldots, n_{N_{\mathrm{rx}}, k}\right]^{\top}$ gathers the circular-symmetric complex Gaussian noise $n_{m, k}$ with zero mean and variance $\sigma_{\mathrm{n}}^{2}$ at the $m$-th receive branch. The transmit symbols are differentially encoded unit-magnitude phase-shift keying (DPSK) symbols, which are generated as follows:

$$
b_{k, u}=a_{k, u} b_{k-1, u}, \quad b_{0, u}=1,
$$


where $a_{k, u}$ are the data symbols drawn from an $M$-ary PSK constellation $\mathcal{M}=\left\{\mathrm{e}^{\mathrm{j} 2 \pi i / M}\right.$ $i=0,1, \ldots, M-1\}$. Due to the PSK signaling, the total received power per user is $\left\|\boldsymbol{h}_{u}\right\|_{2}^{2}$ and the signal-to-noise ratio (SNR) is given as $\left\|\boldsymbol{h}_{u}\right\|_{2}^{2} / \sigma_{\mathrm{n}}^{2}$.

Assuming the channel to be constant during a transmission burst (blockfading channel), noncoherent detection of user $u$ is performed block-wise over a temporal block $R$ of $N_{\mathrm{b} \text { l }}$ time steps given by

$$
\boldsymbol{R}=\left[\boldsymbol{r}_{0}, \cdots, \boldsymbol{r}_{N_{\mathrm{bl}}-1}\right]
$$

and on the basis of the $N_{\mathrm{bl}} \times N_{\mathrm{bl}}$ correlation matrix

$$
Z_{u} \stackrel{\text { def }}{=} R^{\mathrm{H}} \mathcal{W}_{u} R
$$

using a user-specific diagonal weighting matrix

$$
\mathcal{W}_{u} \stackrel{\text { def }}{=} \operatorname{diag}\left(\zeta_{1, u}, \ldots, \zeta_{N_{\mathrm{r} x}, u}\right) .
$$

There are two detection strategies based on (7). Each user can be detected individually using decision-feedback differential detection (DFDD) [11], where decisions are made successively and previous detected symbols are considered. To cope with multi-user interference, DFDD can be combined with noncoherent decision-feedback equalization (DFDD/nDFE) [12], where the average interference of already detected users is subtracted additionally.

Both presented schemes take advantage of the fact that the per-user induced power

$$
P_{m, u} \stackrel{\text { def }}{=} \mathrm{E}\left\{\left|h_{m, u}\right|^{2}\right\}
$$

known as power-space profile (PSP), exhibits a high concentration at some spatial location at the BS. Thus, the users can be separated for detection and $\mathcal{W}_{u}$ in (8) acts as a filter extracting only the relevant entries in $\boldsymbol{R}$ of each user in the spatial domain. The windowing can be numerically optimized (via a global search) to obtain the maximum signal-to-interference-plus-noise ratio (SINR) in (7), when the PSPs in (9) are known [12,35]. However, due to users located in the far-field, $P_{m, u}$ is equally distributed over the receive antennas. Consequently, this demands an alternative solution to redistribute the power, which is presented in the following.

\subsection{Digital Beamspace Preprocessing}

In order to obtain an advantageous power distribution for noncoherent detection, digital beamspace preprocessing is applied, which is commonly used for DOA estimation in the literature [36-40]. To this end, the receiving block $R$ in (6) is modified according to

$$
\hat{\boldsymbol{R}}=\boldsymbol{W}_{\mathrm{BF}}^{\mathrm{H}} \boldsymbol{R}=\left[\hat{\boldsymbol{r}}_{0}, \hat{\boldsymbol{r}}_{1}, \ldots, \hat{\boldsymbol{r}}_{N_{\mathrm{b}}-1}\right],
$$

where

$$
\boldsymbol{W}_{\mathrm{BF}}=\left[\boldsymbol{w}_{1}, \boldsymbol{w}_{2}, \ldots, \boldsymbol{w}_{N_{\mathrm{b}}}\right]
$$

is a $N_{\mathrm{rx}} \times N_{\mathrm{b}}$ beamforming (BF) matrix. $N_{\mathrm{b}}$ determines the number of simultaneous generated beams within a predefined FoV and has to be equal to the number of receive paths or rather BS antennas $N_{r \times}$. The received signals are superimposed using the respective weight vectors $w_{m}$ for the $m$-th receive branch and the resultant spatial (total) power spectrum can be expressed as

$$
\hat{P}_{m}=w_{m}^{\mathrm{H}} \boldsymbol{Q}_{\boldsymbol{r}} \boldsymbol{w}_{m},
$$

where

$$
Q_{r}=\mathrm{E}\left\{\boldsymbol{r}_{k} r_{k}^{\mathrm{H}}\right\}=\mathrm{E}\left\{\boldsymbol{H} \boldsymbol{H}^{\mathrm{H}}\right\}+\sigma_{\mathrm{n}}^{2} \boldsymbol{I}_{N_{\mathrm{rx}}}
$$


denotes the covariance matrix of the initial received signal $\boldsymbol{r}_{k}$. The PSP $\hat{P}_{m, u}$ (spatial per-user power) after digital beamspace preprocessing can be defined as

$$
\hat{P}_{m, u} \stackrel{\text { def }}{=} \mathrm{E}\left\{\left|\boldsymbol{w}_{m}^{\mathrm{H}} \boldsymbol{h}_{u}\right|^{2}\right\}
$$

and the resulting SINR of the $u$-th user at the $m$-th receive branch is given as

$$
\operatorname{SINR}_{m, u}=\frac{\hat{P}_{m, u}}{\boldsymbol{w}_{m}^{\mathrm{H}} \sigma_{\mathrm{n}}^{2} \boldsymbol{w}_{m}+\sum_{v \neq u} \hat{P}_{m, v}} .
$$

The beamforming matrix $\boldsymbol{W}_{\mathrm{BF}}$ should be designed to maximize the SINR in (15) for the respective users and to fulfill the condition

$$
\boldsymbol{W}_{\mathrm{BF}}^{\mathrm{H}} \boldsymbol{W}_{\mathrm{BF}} \approx \boldsymbol{I}_{N_{\mathrm{rx}}}
$$

to ensure the noise to be spatially white. In the following, the composition of $W_{\mathrm{BF}}$ is discussed for a full- and sub-array architecture.

\subsubsection{Full-Array}

Considering a full-array architecture, a first choice of the beamforming matrix is to select $N_{r x}$ steering vectors defined in (2) as the respective columns, i.e.,

$$
\boldsymbol{W}_{\mathrm{BF}, \mathrm{B}}=\left[\boldsymbol{w}_{\mathrm{B}, 1}, \boldsymbol{w}_{\mathrm{B}, 2}, \ldots, \boldsymbol{w}_{\mathrm{B}, \mathrm{N}_{\mathrm{rx}}}\right]
$$

and

$$
\boldsymbol{w}_{\mathrm{B}, m}=\boldsymbol{a}_{N_{\mathrm{rx}}}\left(\phi_{m}\right)=\frac{1}{\sqrt{N_{\mathrm{rx}}}}\left[\begin{array}{c}
1 \\
\mathrm{e}^{-\mathrm{j} k d_{\mathrm{a}} \sin \left(\phi_{m}\right)} \\
\vdots \\
\mathrm{e}^{-\mathrm{j} k\left(N_{\mathrm{rx}}-1\right) d_{\mathrm{a}} \sin \left(\phi_{m}\right)}
\end{array}\right],
$$

where $\phi_{m} \in[-\Phi / 2, \Phi / 2]$ is the corresponding beam direction of the $m$-th receive branch chosen from a given FoV $\Phi$. This method is also known as conventional or Bartlett beamforming (BBF) [36]. It can also be interpreted as an $N_{r x}$-point discrete Fourier transform (DFT), where the weights $w_{\mathrm{B}, m}$ represent a bank of narrowband filters each tuned to the spatial frequency

$$
\theta_{m}=\frac{d_{\mathrm{a}}}{\lambda} \sin \left(\phi_{m}\right)
$$

which is related to the respective angles $\phi_{m}$ or rather the spatial location $m$. A uniform sampling in the frequency domain given a FoV $\Phi$ leads to a spatial frequency spacing

$$
\Delta \theta=\frac{2 d_{\mathrm{a}}}{N_{\mathrm{r} \times} \lambda} \sin (\Phi / 2) \text {. }
$$

Considering the entire visible range $\Phi=\pi$ and the general antenna distance $d_{\mathrm{a}}=\lambda / 2$, the $N_{\mathrm{rx}}$ spatial beams become orthogonal with spacing $\Delta \theta=1 / N_{\mathrm{rx}}$. This corresponds to an angular spacing of $\Delta \phi=\sin ^{-1}(\lambda / D)$, which is a measure of the angular resolution of a uniform linear array with aperture size $D=N_{\mathrm{rx}} d_{\mathrm{a}}$ also known as Rayleigh resolution limit [39]. Strictly speaking, this does not hold for reception at endfire directions, where the resolution drops due to a reduced array aperture size. Furthermore, the FoV is limited by the antenna pattern in practice. Thus, the orthogonality of the beams vanishes when uniformly chosen from a smaller angular range $\Phi<\pi$. Alternatively, the antenna distance $d_{\mathrm{a}}$ could be optimized (increased) for a specific FoV such that $\Delta \theta=1 / N_{\mathrm{rx}}$ holds in (20) in order to restore the orthogonality. 
The resultant spatial power distribution of a plane-wave phase front associated with direction $\phi$ can be expressed as [39]

$$
\begin{aligned}
\left|w_{\mathrm{B}, m}^{\mathrm{H}} \boldsymbol{a}_{N_{\mathrm{rx}}}(\phi)\right|^{2} & =\frac{1}{N_{\mathrm{rx}}^{2}}\left|\sum_{n=1}^{N_{\mathrm{rx}}} \mathrm{e}^{-\mathrm{j} n k d_{\mathrm{a}}\left(\sin (\phi)-\sin \left(\phi_{m}\right)\right)}\right|^{2} \\
& =\frac{1}{N_{\mathrm{rx}}^{2}}\left|\frac{\sin \left(\frac{N_{\mathrm{rx}}}{2} k d_{\mathrm{a}}\left(\sin (\phi)-\sin \left(\phi_{m}\right)\right)\right)}{\sin \left(\frac{1}{2} k d_{\mathrm{a}}\left(\sin (\phi)-\sin \left(\phi_{m}\right)\right)\right)}\right|^{2} \\
& \approx \operatorname{sinc}^{2}\left(\frac{\pi D}{\lambda}\left(\sin (\phi)-\sin \left(\phi_{m}\right)\right)\right),
\end{aligned}
$$

which is approximately a squared sinc-function sampled at the chosen beam directions $\phi_{m}$ of the $m$-th receive branch. Its peak is shifted towards the spatial location, where the direction of the incoming wave matches one of the generated beams, i.e., where $\phi_{m}=\phi$. This is exemplary depicted in Figure 2, where two plane waves separated by two adjacent beams impinge at an array of $N_{r x}=12$ elements considering an FoV of $\Phi=\pi / 2$. Apparently, the signals are not entirely resolvable, since the selected beams are not orthogonal in case of $d_{\mathrm{a}}=\lambda / 2$. This in turn results into (strong) spatial interference. Hence, when the angular separation of the impinging signals is small, the spatial interference rejection capability using Bartlett beamforming is limited.

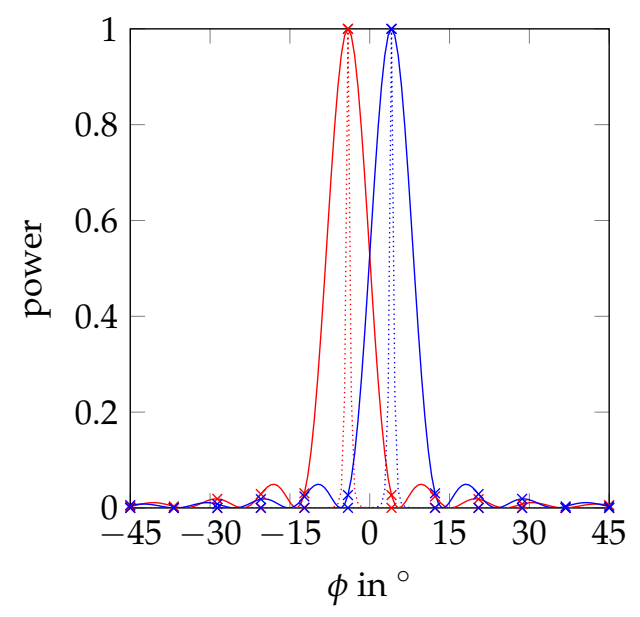

Figure 2. Spatial power distribution at $N_{r x}=12$ receive branches $(x)$ in case of Bartlett beamforming ( - ) and in case of a combination with eigenspace-based post-processing ( $\cdots \cdots \cdots .$. considering two incident waves $(\square / \square)$ and a field of view (FoV) of $\Phi=\pi / 2$.

The optimal solution in terms of spatial filtering and maximizing the SINR is obtained via Capon's method (or a minimum variance distortionless response filter (MVDR)) [36,40,41], where the signal of interest is passed undistorted while the power from all other spatial frequencies and directions is minimized. Based on the knowledge of the covariance matrix in (13), this data-dependent approach leads to a higher resolution and thus power focusing capability than the conventional beamforming. However, mismatches in the array response due to multi-path propagation and imprecise knowledge of the covariance matrix, which has to be estimated in practice, lead to a severe performance degradation [40]. The same applies to subspace-based methods such as multiple signal classification (MUSIC) [36,42] or rotational invariance techniques (ESPRIT) [43], which offer high resolution capabilities. 
Alternatively, a combination of Bartlett and eigenspace-based processing [44], denoted as Bartlett-eigenspace beamforming (BEBF) in the following, can provide a more robust approach against aforementioned issues. The corresponding overall weighting matrix can be expressed as

$$
\boldsymbol{W}_{\mathrm{BF}, \mathrm{BE}}=\boldsymbol{W}_{\mathrm{BF}, \mathrm{B}} \boldsymbol{W}_{\mathrm{BF}, \mathrm{E}},
$$

where

$$
\boldsymbol{W}_{\mathrm{BF}, \mathrm{E}}=\left[\boldsymbol{w}_{\mathrm{E}, 1}, \boldsymbol{w}_{\mathrm{E}, 2}, \ldots, \boldsymbol{w}_{\mathrm{E}, N_{\mathrm{rx}}}\right]
$$

denotes the eigenspace-based beamforming matrix. In an initial step, conventional beamforming is performed for spatial prefiltering according to

$$
\overline{\boldsymbol{R}}=\boldsymbol{W}_{\mathrm{BF}, \mathrm{B}}^{\mathrm{H}} \boldsymbol{R}=\left[\overline{\boldsymbol{r}}_{0}, \overline{\boldsymbol{r}}_{1}, \ldots, \overline{\boldsymbol{r}}_{\mathrm{N}_{\mathrm{bl}}-1}\right] .
$$

Due to the beamspace transformation, the array response vector changes to

$$
\overline{\boldsymbol{a}}_{N_{\mathrm{rx}}}\left(\phi_{m}\right)=\boldsymbol{W}_{\mathrm{BF}, \mathrm{B}}^{\mathrm{H}} \boldsymbol{a}_{N_{\mathrm{rx}}}\left(\phi_{m}\right) \text {. }
$$

The covariance matrix of (24) can be computed via its sample estimate and an eigenvalue decomposition is applied as follows:

$$
\tilde{Q}_{\bar{r}}=1 / N_{\mathrm{bl}} \bar{R} \bar{R}^{\mathrm{H}}=V_{s} \boldsymbol{\Lambda}_{s} \boldsymbol{V}_{s}^{\mathrm{H}}+\boldsymbol{V}_{n} \boldsymbol{\Lambda}_{n} \boldsymbol{V}_{n}^{\mathrm{H}},
$$

where the diagonal matrices $\Lambda_{s}$ and $\Lambda_{n}$ contain the $N_{\mathrm{s}}$ largest (signal-subspace) and the $N_{\mathrm{rx}}-N_{\mathrm{s}}$ smallest (noise-subspace) eigenvalues, respectively. $V_{s}$ and $V_{n}$ denote the corresponding eigenvectors. The respective weight vectors in (23) for eigenspace-based post-processing are composed according to

$$
\boldsymbol{w}_{\mathrm{E}, m}=\frac{\tilde{\boldsymbol{Q}}_{\overline{\boldsymbol{r}}}^{-1} \boldsymbol{V}_{s} \boldsymbol{V}_{s}^{\mathrm{H}} \overline{\boldsymbol{a}}_{N_{\mathrm{rx}}}\left(\phi_{m}\right)}{\overline{\boldsymbol{a}}_{N_{\mathrm{rx}}}^{\mathrm{H}}\left(\phi_{m}\right) \tilde{Q}_{\overline{\boldsymbol{r}}}^{-1} \overline{\boldsymbol{a}}_{N_{\mathrm{rx}}}\left(\phi_{m}\right)} .
$$

Comparing the resultant spatial power distribution to conventional beamforming as illustrated in Figure 2, the additional eigenspace-based post-processing restores the orthogonality of the selected beams and achieves a higher resolution as expected. This provides more flexibility since the beams do not need to be selected from a regular grid anymore. Most importantly, the energy of the incoming wave is focused almost entirely to one receive branch reducing the spatial interference. Noteworthy, this approach is more computational complex than conventional beamforming, which can be implemented via a fast Fourier transform (FFT) later on. Moreover, the knowledge of the number of sources $N_{\mathrm{s}}$ is required, i.e., the number of users $N_{\mathrm{u}}$, and this method can suffer from instabilities when performing the inverse of the estimated covariance matrix. Diagonal loading can counteract this issue [40].

\subsubsection{Sub-Array}

All above mentioned beamforming schemes for a full-array architecture can be applied analogously to the sub-array architecture. Instead of $N_{r x}$ beams according to the number of receive antennas/branches, the number of beams is limited to the per-sub-array elements $N_{\text {rx,sub }}=N_{\text {rx }} / N_{\text {sub }}$ and, when applied, the eigenspace-based post-processing is performed separately at each sub-array. The beam directions can be chosen from a different set and/or angular sector since each sub-array can process the incoming signals individually. However, the condition in (16) might be not fulfilled in this case. Therefore, the same set of beams is used per sub-array selected from a predefined FoV $\Phi$. The corresponding weight matrix of the $l$-th sub-array is

$$
\boldsymbol{W}_{\mathrm{BF}_{\mathrm{sub}, l}}=\left[\boldsymbol{w}_{1}, \boldsymbol{w}_{2}, \ldots, \boldsymbol{w}_{N_{\mathrm{rx}, \mathrm{sub}}}\right]
$$


and the total beamforming matrix is constructed via

$$
W_{\mathrm{BF}}=\operatorname{diag}\left(W_{\mathrm{BF}_{\mathrm{sub}}, 1}, \ldots, W_{\mathrm{BF}_{\mathrm{sub}}, \mathrm{N}_{\mathrm{sub}}}\right) \text {. }
$$

\section{Numerical Results}

Numerical simulations are conducted for an $N_{\mathrm{u}}=3$ user scenario and a BS employing a uniform linear array of $N_{\mathrm{rx}}=128$ directional patch antennas [15] with inter-element distance $d_{\mathrm{a}}=\lambda / 2$. The FoV of the BS, where the incoming signals can be processed from, is assumed to be $\Phi=\pi / 2$. The users equipped with single omni-directional antennas are located under a given angle $\phi_{u}$ in the far-field of the BS as illustrated in Figure 3. In the following, users 1 and 3 are positioned at a fixed angle of $\phi_{1}=-30^{\circ}$ and $\phi_{3}=30^{\circ}$, respectively.

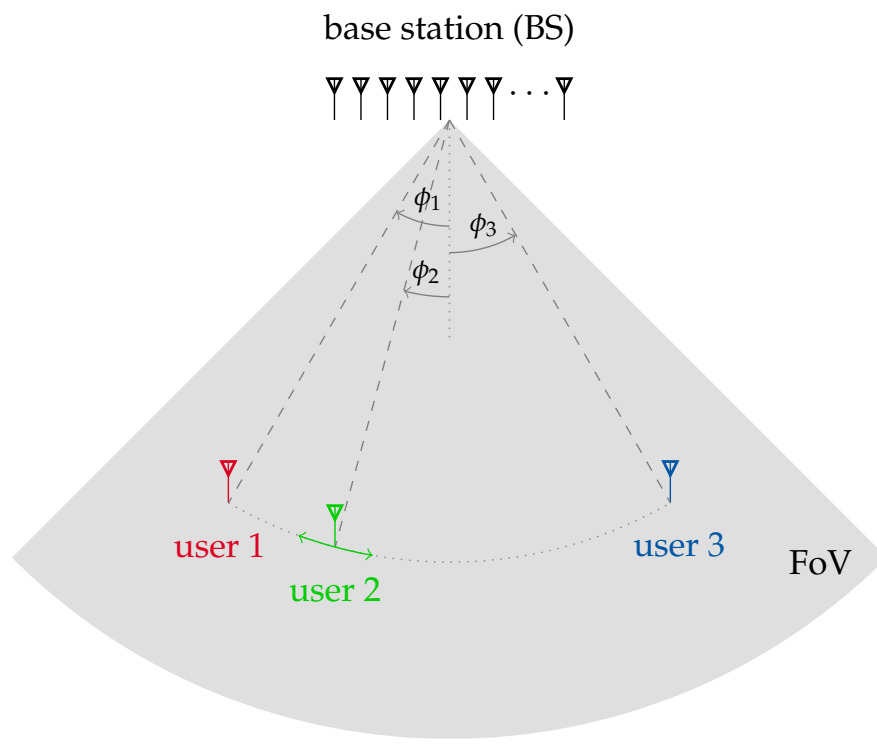

Figure 3. User and base station (BS) arrangement to evaluate the noncoherent massive MIMO system with digital beamspace preprocessing.

A propagation channel is considered, where both LOS and multi-path propagation are taken into account and its respective power ratio is set according to (3). The multi-path components of each user, which are in total $N_{p}=3$, impinge at the BS having an angular spread with standard deviation $\sigma_{\mathrm{s}}$ from the nominal angle $\phi_{u}$. The respective channel matrices from the cluster-based channel model are normalized in such a way that $\|\boldsymbol{H}\|_{\mathrm{F}}^{2}=N_{\mathrm{u}}$. This power control ensures that the total induced sum power at the BS is equal to the number of users and the difference in channel attenuation between the users remain. If the total receive power of the users is equal, the total SNR per user reads $1 / \sigma_{n}^{2}$, which is used for setting the noise power $\sigma_{\mathrm{n}}^{2}$ at each antenna branch.

The performance measure is the symbol error rate (SER), which is evaluated for noncoherent detection using DFDD/nDFE with (and without) digital beamspace preprocessing. To this end, transmit symbols from a quaternary DPSK constellation and a transmission burst of length $N_{\mathrm{bl}}=201$ are considered. The SER is averaged over $N_{\mathrm{ch}}=50,000$ channel realizations acquired from the adapted COST 2100 channel model.

The system configuration and the parameterization of both propagation channel and noncoherent detection are summarized in Table 1. 
Table 1. System configuration and parameterization of propagation channel and noncoherent detection.

\begin{tabular}{cc}
\hline \multicolumn{2}{c}{ System Configuration } \\
\hline number of users $N_{\mathrm{u}}$ & 3 \\
number of BS antennas $N_{\mathrm{rx}}$ & 128 \\
BS antenna spacing $d_{\mathrm{a}}$ & $\lambda / 2$ \\
FoV $\Phi$ & $\pi / 2$ \\
user antenna type & omni-directional \\
BS antenna type & patch $[15]$ \\
angle $\phi_{u}$ of user & $\phi_{1}=-30^{\circ}, \phi_{2}, \phi_{3}=30^{\circ}$ \\
\hline Noncoherent Detection \\
\hline modulation alphabet \\
block length $N_{\mathrm{bl}}$ \\
\hline Propagation Channel \\
\hline cluster types & 4 -ary DPSK \\
\hline mumber of multi-path components $N_{p}$ & local \\
angular spread $\sigma_{\mathrm{s}}$ at BS & 3 \\
LOS-to-MPC ratio (LMR) & variable \\
nom & variable \\
number of different channel realizations $N_{\mathrm{ch}}$ & 50,000 \\
channel normalization (power control) & $\|\boldsymbol{H}\|_{\mathrm{F}}^{2}=N_{\mathrm{u}}$ \\
\hline
\end{tabular}

\subsection{Full-Array vs. Sub-Array Architecture}

First, the full- and sub-array architectures are investigated. To this end, a dominant LOS channel is considered, where the power of the LOS component is significantly larger than the sum power of the multi-path components originating from local clusters around the users. The LMR is set to $10 \mathrm{~dB}$ and the spatial spread of the multi-path components is $\sigma_{\mathrm{s}}=2^{\circ}$. The PSPs of the users are assumed to be perfectly known and the beamforming weights are chosen optimally, i.e., the beams are evenly distributed in the given $\operatorname{FoV} \Phi=\pi / 2$, whereby one of the generated beams is perfectly aligned to a respective user. For comparison, the SER performance of coherent detection via Bell Laboratories layered space-time (BLAST) $[45,46]$ with perfect channel knowledge is included in the following considerations.

Applying the digital beamspace preprocessing, the induced power of the users at the BS, which has been uniformly distributed over the receive antennas due the to far-field location, is spatially redistributed and focused onto a smaller subset of the $N_{r x}$ receive branches.

First, regarding the full-array architecture, the resulting PSPs show a very narrow peak as depicted in Figure $4 \mathrm{a}$, where user 2 is located at boresight $\left(\phi_{2}=0^{\circ}\right)$. In case of conventional beamforming, the PSPs follow a squared sinc function, which is minimally broadened due to the low-power multi-path components. A combination with eigenspace-based beamforming yields even narrower power peaks, where most of the user power is concentrated at one receive branch. A difference in the total power of the users can be observed, which is due to a loss in received power for non-boresight users since directional patch antennas are employed at the BS.

The SER performance as function of the SNR is illustrated in Figure $4 \mathrm{~b}$ when user 2 is again at the boresight location. While noncoherent detection fails without beamspace preprocessing due to uniform PSPs, the spatial redistribution of the user power in the digital domain via beamforming allows almost identical performance compared to the coherent detection with perfect channel knowledge. Here, no performance gains are observed when eigenspace-based processing is applied in addition. The worse performance of the users 1 and 3 is again related to the power loss using directional antennas.

Analyzing the SER as function of the angular position $\phi_{2}$ of user 2 (see Figure 4c), the performance of the noncoherent detection scheme deteriorates when approaching one of the other users due to the overlapping of the respective PSPs. Here, additional eigenspace-based beamforming exhibits a significant improvement in terms of user separability since the digital redistributed user power is 
almost located at one receive branch. Noteworthy, coherent detection using BLAST exhibits a minor degradation in performance when the users are exactly at the same angular position.

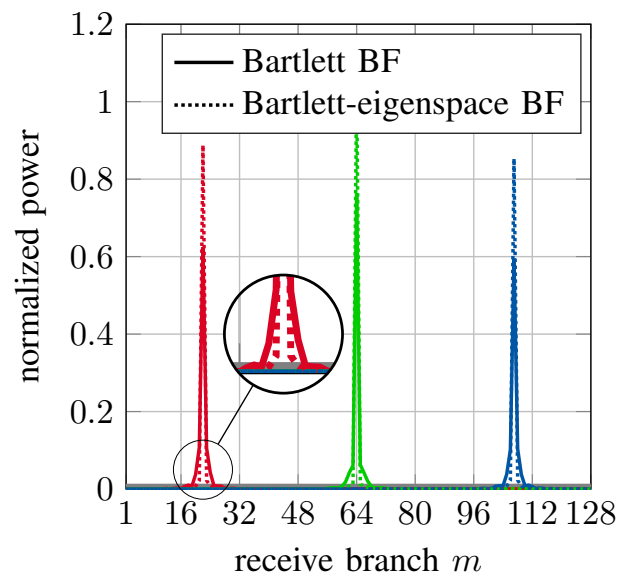

(a) Power-space profile $\hat{P}_{m, u}$ for user 2 at boresight $\left(\phi_{2}=0^{\circ}\right)$.

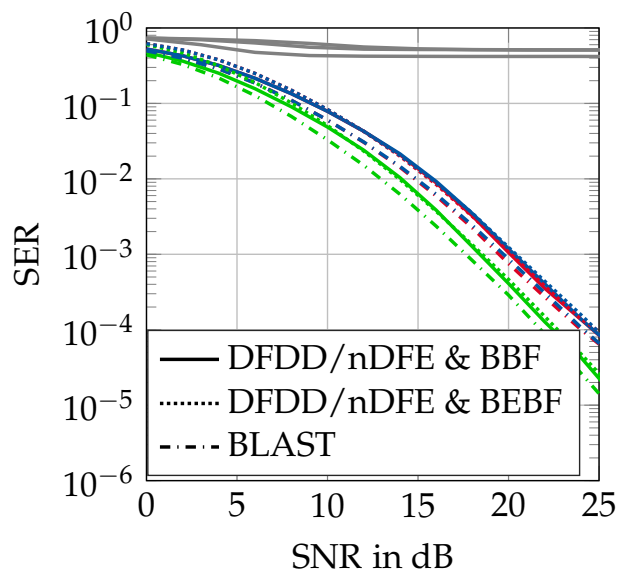

(b) Symbol error rate vs.

signal-to-noise ratio for user 2 at boresight $\left(\phi_{2}=0^{\circ}\right)$.

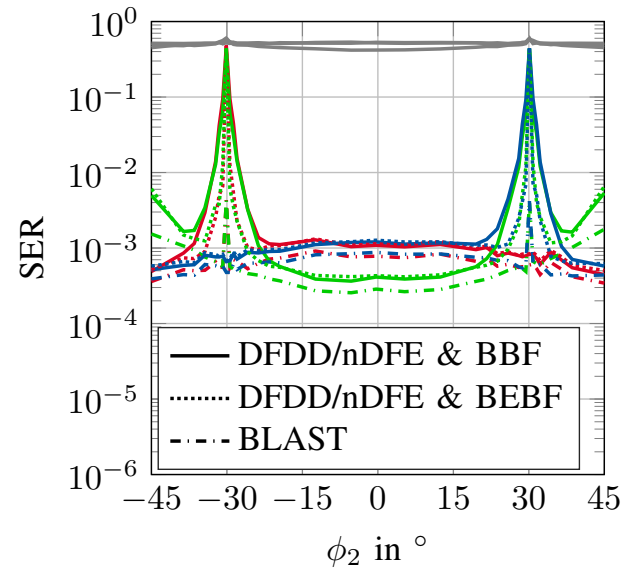

(c) Symbol error rate vs. angle $\phi_{2}$ of user 2 for $\mathrm{SNR}=20 \mathrm{~dB}$.

Figure 4. Full-array architecture: Power-space profile and symbol error rate performance. Colors correspond to users: user $1(\square)$, user $2(\square)$, user $3(\square)$. Gray plots $(\square)$ represent results without digital beamspace preprocessing for each user.

Next, the sub-array architecture is considered allowing a low-complexity LO distribution network since each sub-array can employ its own LO. To this end, the antenna array of the BS is splitted into $N_{\text {sub }}=8$ sub-arrays each comprising $N_{r x, s u b}=16$ antennas. Consequently, 16 different spatial beams can be generated, which are assumed to be the same for each sub-array. This leads to a periodic pattern of the PSPs as depicted in Figure 5a. The SER as function of the SNR remains almost unchanged when compared to the full-array architecture using Bartlett beamforming (see Figure 5b). A saturation is observed at high SNR, where the residual inter-user interference due to non-orthogonal beams begins to dominate, deteriorating the performance. A slight performance degradation is observed for Bartlett-eigenspace beamforming. Analyzing the SER vs. the angle $\phi_{2}$, the user separability is diminished due to a loss in angular resolution (see Figure 5c). Thus, closely spaced users cannot be separated anymore as compared to the full-array case since the individual PSPs of the users already start to overlap at larger angular distance. Here, eigenspace-based processing reveals again a superior performance than just conventional beamforming. 


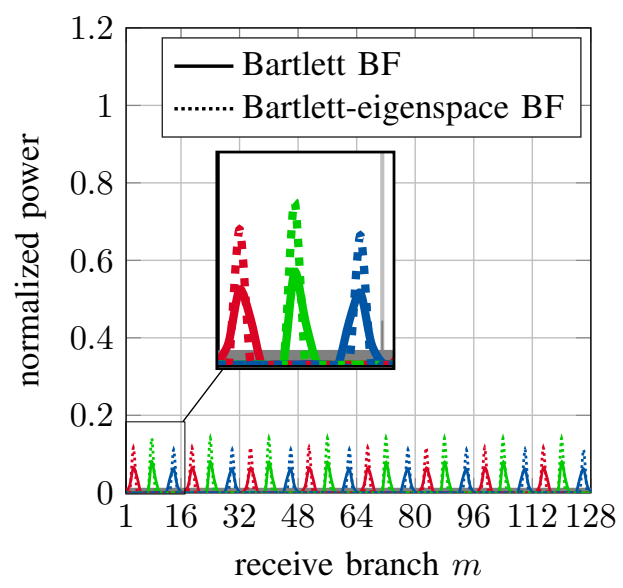

(a) Power-space profile $\hat{P}_{m, u}$ for user 2 at boresight $\left(\phi_{2}=0^{\circ}\right)$.

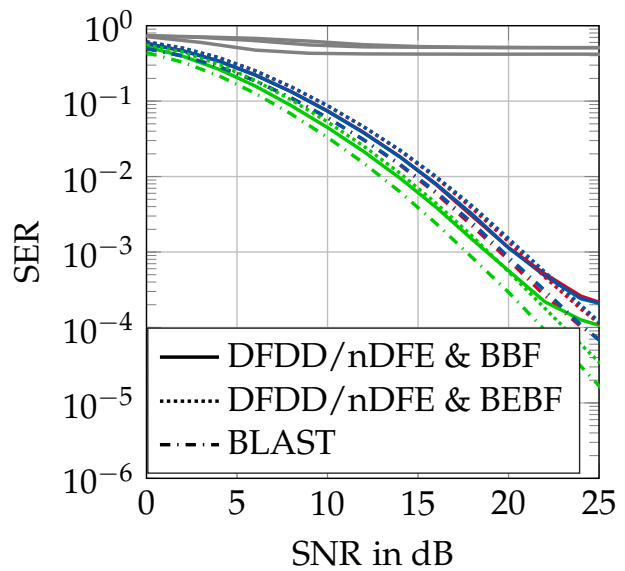

(b) Symbol error rate vs.

signal-to-noise ratio for user 2 at boresight $\left(\phi_{2}=0^{\circ}\right)$.

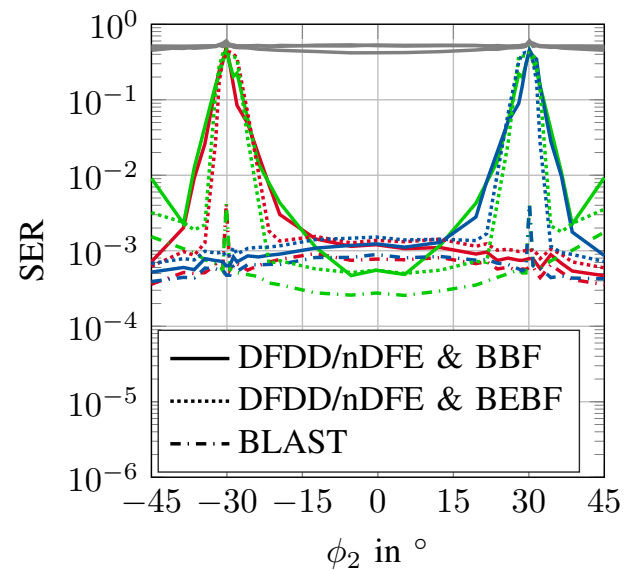

(c) Symbol error rate vs. angle $\phi_{2}$ of user 2 for $\mathrm{SNR}=20 \mathrm{~dB}$.

Figure 5. Sub-array architecture of $N_{\text {sub }}=8$ and $N_{\text {rx,sub }}=16$ : Power-space profile and symbol error rate performance. Colors correspond to users: user $1(\square)$, user $2(\square)$, user $3(\square)$. Gray plots $(\square)$ represent results without digital beamspace preprocessing for each user.

Results of different sub-array configurations are illustrated in Figure 6 considering various angular positions of user 2 . The smaller the number of antennas per sub-array $N_{\mathrm{rx}, \mathrm{sub}}$, the smaller the angular resolution and thus the user separability. At small $N_{r x, s u b}$, the conventional beamforming suffers from performance variations, which originates from strong inter-user interference caused by non-orthogonal beams. On the contrary, eigenspace post-processing offers a better user separation at all times. 


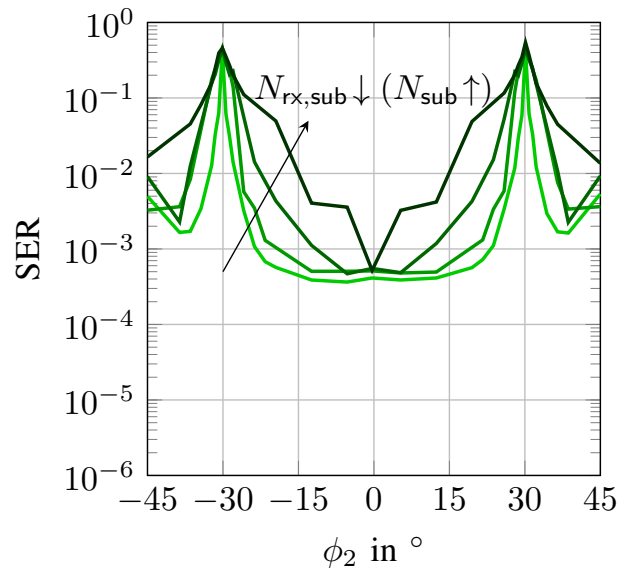

(a) Bartlett BF.

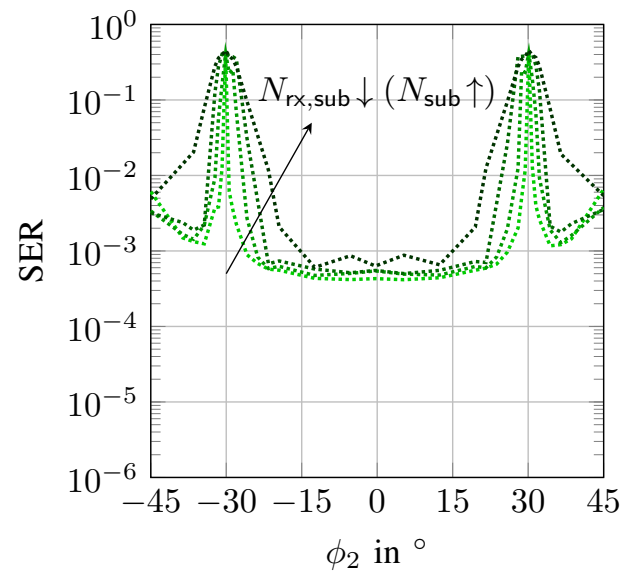

(b) Bartlett-eigenspace BF.

Figure 6. Symbol error rate vs. angle $\phi_{2}$ of user 2 for the full-array system ( $\square$ ) and for different sub-array configurations $\left(N_{\text {sub }}=4\right.$ and $N_{\text {rx,sub }}=32(\square), N_{\text {sub }}=8$ and $N_{\text {rx,sub }}=16(\square), N_{\text {sub }}=16$ and $N_{\text {rx,sub }}=8(\square)$.

\subsection{Influence of Propagation Channel}

In the following, the influence of the propagation channel is analyzed. To this purpose, the dominance of the LOS is varied by means of the LMR and the spatial spread $\sigma_{\mathrm{s}}$ of the multi-path components is modified. The analysis is restricted to the full-array architecture (observations apply to the sub-array in the same manner). The stronger the LOS component compared to the multi-path components, the narrower are the individual PSPs enhancing the angular user separation for both conventional beamforming and the combination with eigenspace-based processing due to lower inter-user interference (see Figure 7). At the same time, higher channel correlations deteriorate the overall performance. Increasing the spatial spread $\sigma_{\mathrm{s}}$, the multi-path components become more relevant and cause a broadening of the PSPs in case of conventional beamforming. Consequently, a higher inter-user interference occur and the user separability is worse in these scenarios (see Figure 8). On the contrary, additional eigenspace-based processing is insensitive to higher spatial spreads regarding the user separation and exhibits a performance improvement due to lower channel correlations.

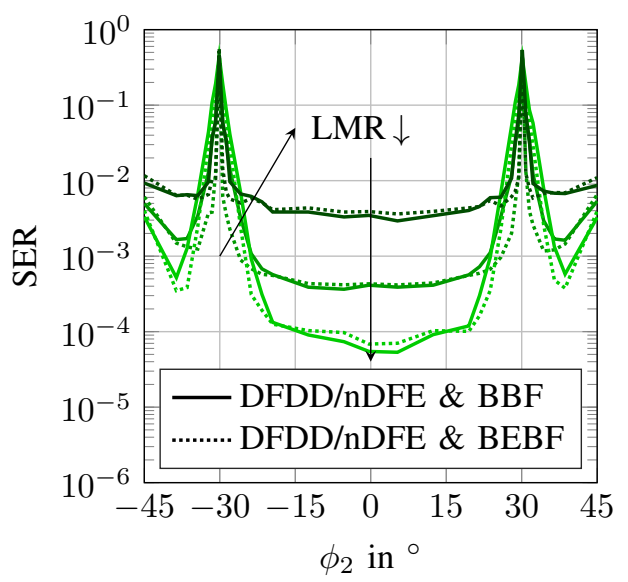

Figure 7. Symbol error rate vs. angle $\phi_{2}$ of user 2 at different LMR considering the full-array architecture: $\mathrm{LMR}=0 \mathrm{~dB}(\square), \mathrm{LMR}=10 \mathrm{~dB}(\square), \mathrm{LMR}=20 \mathrm{~dB}(\square)$. 


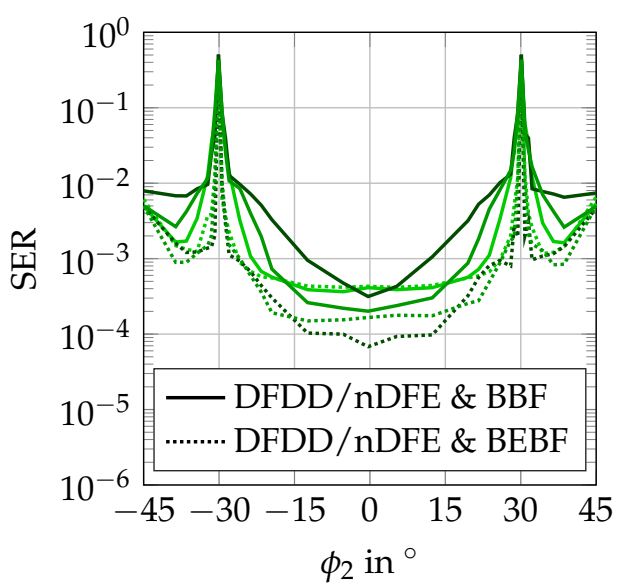

Figure 8. Symbol error rate vs. angle $\phi_{2}$ of user 2 at different spatial spread $\sigma_{\mathrm{s}}$ considering the full-array architecture: $\sigma_{\mathrm{s}}=2^{\circ}(\square), \sigma_{\mathrm{s}}=4^{\circ}(\square), \sigma_{\mathrm{s}}=8^{\circ}(\square)$.

In summary, noncoherent detection with beamspace preprocessing performs best when channel correlations are low, i.e., multi-path propagation is prevailing and the multi-path components impinge at a large angular spread at the BS. However, in order to achieve best performance in terms of user separability, a dominant LOS channel needs to be present, where the standard deviation of the multi-path components from the nominal LOS angle is preferably small at least for conventional beamforming. This allows to accommodate a large number of users. Ideally, one generated beam or rather receive branch can serve one user. Thus, the upper bound is $N_{r x}$ in the full-array and $N_{r x, s u b}$ in the sub-array case. However, this limit might be reached in pure LOS channels or/and for eigenspace-based beamforming, where the orthogonality of the beams is given as compared to conventional beamforming.

\section{Conclusions}

In this paper, the application of digital beamspace preprocessing to a massive MIMO system is assessed, where noncoherent detection is performed in the uplink. First, the fundamentals and the requirements are discussed when both concepts are treated jointly and when a full-array or a sub-array architecture is considered. Then, the symbol error rate performance is analyzed for a three-user scenario on the basis of a cluster-based channel model capturing its principal behavior under physically accurate conditions. It is demonstrated that digital beamspace preprocessing transforms the unsuitable uniform PSPs, when the users are located in the far-field of the BS, into PSPs with a narrow peak located at the receive branch associated with the corresponding angle of arrival of the incoming wave. Hence, the users can be spatially separated when the signals impinge at the BS from sufficiently different angular directions enabling again the noncoherent detection scheme. An improved performance in terms of angular user separability is achieved when the conventional processing is extended by means of eigenspace-based beamforming. At the costs of higher computational complexity, this procedure reduces the inter-user interference in case of closely spaced users. Regarding a sub-array configuration, the PSPs exhibit a periodic pattern and the angular user separation is less due the reduced aperture size. Furthermore, a small performance degradation is observed.

The characteristics of the propagation channel can have a large impact on the system performance. Best results in terms of user separability are obtained in dominant LOS channels, which allows an accommodation of a large number of users. On the contrary, better overall SER performance is achieved in multi-path scenarios since the channel correlations are lower. However, a higher level of inter-user interference can occur for larger spatial spreads of the multi-path components reducing the total number of users, which can be served optimally.

Generally, the knowledge of the PSPs for noncoherent detection are unknown in practice. However, the estimation is straightforward when the angular position of the users is known beforehand. Otherwise, the common known concept of DOA estimation can be employed extracting the respective 
angular directions via a peak search in the angular spectrum. This works particularly in dominant LOS channels. The extracted knowledge of the angular position can also be used for the downlink transmission afterwards.

Comparing the results to coherent detection with perfect channel knowledge, the performance gap is minimal. However, the estimation overhead and potential channel estimation errors are not taken into account, which severely degrades its performance. When considered, the presented combination of the noncoherent detection scheme and digital beamspace preprocessing outperforms the CSI-based detection.

Author Contributions: Conceptualization, S.B.; methodology, S.B.; software, S.B.; validation, S.B.; formal analysis, S.B.; investigation, S.B.; resources, C.W.; data curation, S.B.; writing-original draft preparation, S.B.; writing-review and editing, S.B. and C.W.; visualization, S.B.; supervision, C.W.; project administration, C.W.; funding acquisition, C.W. All authors have read and agreed to the published version of the manuscript.

Funding: This research was funded by the Deutsche Forschungsgemeinschaft (DFG, German Research Foundation) grant number WA 3506/5-1.

Conflicts of Interest: The authors declare no conflict of interest. The funders had no role in the design of the study; in the collection, analyses, or interpretation of data; in the writing of the manuscript, or in the decision to publish the results.

\section{References}

1. Gupta, A.; Jha, R.K. A Survey of 5G Network: Architecture and Emerging Technologies. IEEE Access 2015, 3, 1206-1232, [CrossRef]

2. Marzetta, T.L. Noncooperative Cellular Wireless with Unlimited Numbers of Base Station Antennas. IEEE Trans. Wirel. Commun. 2010, 9, 3590-3600, [CrossRef]

3. Larsson, E.G.; Edfors, O.; Tufvesson, F.; Marzetta, T.L. Massive MIMO for Next Generation Wireless Systems. IEEE Commun. Mag. 2014, 52, 186-195, [CrossRef]

4. Rusek, F.; Persson, D.; Lau, B.K.; Larsson, E.G.; Marzetta, T.L.; Edfors, O.; Tufvesson, F. Scaling Up MIMO: Opportunities and Challenges with Very Large Arrays. IEEE Signal Process. Mag. 2013, 30, 40-60, [CrossRef]

5. Andrews, J.G.; Buzzi, S.; Choi, W.; Hanly, S.V.; Lozano, A.; Soong, A.C.K.; Zhang, J.C. What Will 5G Be? IEEE J. Sel. Areas Commun. 2014, 32, 1065-1082, [CrossRef]

6. Lu, L.; Li, G.Y.; Swindlehurst, A.L.; Ashikhmin, A.; Zhang, R. An Overview of Massive MIMO: Benefits and Challenges. IEEE J. Sel. Top. Signal Process. 2014, 8, 742-758, [CrossRef]

7. Elijah, O.; Leow, C.Y.; Rahman, T.A.; Nunoo, S.; Iliya, S.Z. A Comprehensive Survey of Pilot Contamination in Massive MIMO-5G System. IEEE Commun. Surv. Tutor. 2016, 18, 905-923, [CrossRef]

8. Stojanovic, M.; Proakis, J.; Catipovic, J. Analysis of the Impact of Channel Estimation Errors on the Performance of a Decision-Feedback Equalizer in Fading Multipath Channels. IEEE Trans. Commun. 1995, 43, 877-886, [CrossRef]

9. Peng, W.; Adachi, F.; Ma, S.; Wang, J.; Ng, T.S. Effects of Channel Estimation Errors on V-BLAST Detection. In Proceedings of the IEEE Global Telecommunications Conference (GLOBECOM), New Orleans, LA, USA, 30 November-4 December 2008, [CrossRef]

10. Marzetta, T.L.; Larsson, E.G.; Yang, H. Fundamentals of Massive MIMO; Cambridge University Press: Cambridge, UK, 2016.

11. Schenk, A.; Fischer, R.F.H. Noncoherent Detection in Massive MIMO Systems. In Proceedings of the 17th International ITG Workshop on Smart Antennas (WSA), Stuttgart, Germany, 13-14 March 2013; pp. 1-8.

12. Fischer, R.F.H.; Bense, M. Noncoherent Decision-Feedback Equalization in Massive MIMO Systems. In Proceedings of the International Zurich Seminar on Communications (IZS), Zurich, Switzerland, 26-28 February 2014; pp. 112-115, [CrossRef]

13. Fischer, R.F.H.; Bense, M.; Stierstorfer, C. Noncoherent Joint Decision-Feedback Detection in Multi-User Massive MIMO Systems. In Proceedings of the 18th International ITG Workshop on Smart Antennas (WSA), Erlangen, Germany, 12-13 March 2014; pp. 1-8.

14. Yammine, G.; Fischer, R.F.H. Feedback-Aware Noncoherent Receivers for Massive MIMO Systems. In Proceedings of the 24th International ITG Workshop on Smart Antennas (WSA), Hamburg, Germany, 18-20 February 2020; pp. 1-6. 
15. Bucher, S.; Yammine, G.; Fischer, R.F.H.; Waldschmidt, C. Influence of Channel Parameters on Noncoherent Massive MIMO Systems. In Proceedings of the 22nd International ITG Workshop on Smart Antennas (WSA), Bochum, Germany, 14-16 March 2018.

16. Bucher, S.; Ragab, A.N.; Yammine, G.; Fischer, R.F.H.; Waldschmidt, C. Antenna Design for Noncoherent Massive MIMO Systems. In Proceedings of the 15th International Symposium on Wireless Communication Systems (ISWCS), Lisbon, Portugal, 28-31 August 2018, [CrossRef]

17. Bucher, S.; Yammine, G.; Fischer, R.F.H.; Waldschmidt, C. On the Impact of Hardware Impairments in Noncoherent Massive MIMO Systems. In Proceedings of the 24th International ITG Workshop on Smart Antennas (WSA), Hamburg, Germany, 18-20 February 2020.

18. Bense, M.; Weigel, R. Channel Measurements for the Evaluation of Noncoherent Massive MIMO Systems. In Proceedings of the 47th European Microwave Conference (EuMC), Nuremberg, Germany, 10-12 October 2017, [CrossRef]

19. Yammine, G.; Bucher, S.; Fischer, R.F.H. Noncoherent Detection for an EM-Lens-Enabled Massive MIMO System. In Proceedings of the International ITG Conference on Systems, Communication and Coding (SCC), Rostock, Germany, 11-14 February 2019.

20. Bucher, S.; Yammine, G.; Fischer, R.F.H.; Waldschmidt, C. A Noncoherent Massive MIMO System Employing Beamspace Techniques. IEEE Trans. Veh. Technol. 2019, 68, 11052-11063, [CrossRef]

21. Zeng, Y.; Zhang, R.; Chen, Z.N. Electromagnetic Lens-Focusing Antenna Enabled Massive MIMO: Performance Improvement and Cost Reduction. IEEE J. Sel. Areas Commun. 2014, 32, 1194-1206, [CrossRef]

22. Zeng, Y.; Zhang, R. Millimeter Wave MIMO With Lens Antenna Array: A New Path Division Multiplexing Paradigm. IEEE Trans. Commun. 2016, 64, 1557-1571, [CrossRef]

23. Zeng, Y.; Yang, L.; Zhang, R. Multi-User Millimeter Wave MIMO With Full-Dimensional Lens Antenna Array. IEEE Trans. Wirel. Commun. 2018, 17, 2800-2814, [CrossRef]

24. Zeng, Y.; Zhang, R. Cost-Effective Millimeter-Wave Communications with Lens Antenna Array. IEEE Wirel. Commun. 2017, 24, 81-87, [CrossRef]

25. Kwon, T.; Lim, Y.; Chae, C. Limited Channel Feedback for RF Lens Antenna Based Massive MIMO Systems. In Proceedings of the International Conference on Computing, Networking and Communications (ICNC), Kauai, HI, USA, 15-18 February 2015; pp. 6-10, [CrossRef]

26. Kwon, T.; Lim, Y.G.; Min, B.W.; Chae, C.B. RF Lens-Embedded Massive MIMO Systems: Fabrication Issues and Codebook Design. IEEE Trans. Microw. Theory Tech. 2016, 64, 2256-2271, [CrossRef]

27. Sayeed, A.; Behdad, N. Continuous Aperture Phased MIMO: Basic Theory and Applications. In Proceedings of the 48th Annual Allerton Conference on Communication, Control, and Computing (Allerton), Monticello, IL, USA, 29 Septembet-1 October 2010; pp. 1196-1203, [CrossRef]

28. Brady, J.; Behdad, N.; Sayeed, A.M. Beamspace MIMO for Millimeter-Wave Communications: System Architecture, Modeling, Analysis, and Measurements. IEEE Trans. Antennas Propag. 2013, 61, 3814-3827, [CrossRef]

29. Amadori, P.V.; Masouros, C. Low RF-Complexity Millimeter-Wave Beamspace-MIMO Systems by Beam Selection. IEEE Trans. Commun. 2015, 63, 2212-2223, [CrossRef]

30. Huang, W.; Huang, Y.; Zeng, Y.; Yang, L. Wideband Millimeter Wave Communication With Lens Antenna Array: Joint Beamforming and Antenna Selection With Group Sparse Optimization. IEEE Trans. Wirel. Commun. 2018, 17, 6575-6589, [CrossRef]

31. Shen, W.; Bu, X.; Gao, X.; Xing, C.; Hanzo, L. Beamspace Precoding and Beam Selection for Wideband Millimeter-Wave MIMO Relying on Lens Antenna Arrays. IEEE Trans. Signal Process. 2019, 67, 6301-6313, [CrossRef]

32. Balanis, C.A. Antenna Theory: Analysis and Design, 3rd ed.; John Wiley \& Sons: Hoboken, NJ, USA, 2005.

33. Liu, L.; Oestges, C.; Poutanen, J.; Haneda, K.; Vainikainen, P.; Quitin, F.; Tufvesson, F.; Doncker, P.D. The COST 2100 MIMO Channel Model. IEEE Wirel. Commun. 2012, 19, 92-99, [CrossRef]

34. Verdone, R.; Zanella, A. Pervasive Mobile and Ambient Wireless Communications; Springer: London, UK, 2012, [CrossRef]

35. Yammine, G.; Fischer, R.F.H.; Waldschmidt, C. On the Influence of the Antenna Pattern in Noncoherent Massive MIMO Systems. In Proceedings of the International Symposium on Wireless Communication Systems (ISWCS), Brussels, Belgium, 25-28 August 2015; pp. 391-395, [CrossRef] 
36. Krim, H.; Viberg, M. Two Decades of Array Signal Processing Research: The Parametric Approach. IEEE Signal Process. Mag. 1996, 13, 67-94, [CrossRef]

37. Veen, B.V.; Buckley, K. Beamforming: A Versatile Approach to Spatial Filtering. IEEE ASSP Mag. 1988, 5, 4-24, [CrossRef]

38. Manolakis, D.G.; Ingle, V.K.; Kogon, S.M. Statistical and Adaptive Signal Processing: Spectral Estimation, Signal Modeling, Adaptive Filtering and Array Processing; Artech House: Norwood, MA, USA, 2005.

39. Van Trees, H.L. Optimum Array Processing; Wiley-Blackwell: Hoboken, NJ, USA, 2002.

40. Li, J.; Stoica, P. Robust Adaptive Beamforming; John Wiley \& Sons, Inc.: Hoboken, NJ, USA, 2005, [CrossRef]

41. Capon, J. High-Resolution Frequency-Wavenumber Spectrum Analysis. Proc. IEEE 1969, 57, 1408-1418, [CrossRef]

42. Schmidt, R. Multiple Emitter Location and Signal Parameter Estimation. IEEE Trans. Antennas Propag. 1986, 34, 276-280, [CrossRef]

43. Roy, R.; Kailath, T. ESPRIT-Estimation of Signal Parameters via Rotational Invariance Techniques. IEEE Trans. Acoust. Speech Signal Process. 1989, 37, 984-995, [CrossRef]

44. Yu, J.L.; Yeh, C.C. Generalized Eigenspace-Based Beamformers. IEEE Trans. Signal Process. 1995, 43, 2453-2461, [CrossRef]

45. Foschini, G.J.; Chizhik, D.; Gans, M.J.; Papadias, C.; Valenzuela, R.A. Analysis and Performance of Some Basic Space-Time Architectures. IEEE J. Sel. Areas Commun. 2003, 21, 303-320, [CrossRef]

46. Del Re, E.; Morosi, S.; Marabissi, D.; Mucchi, L.; Pierucci, L.; Ronga, L.S. Reconfigurable Antenna for Future Wireless Communication Systems. Wirel. Pers. Commun. 2007, 42, 405-430, [CrossRef]

Publisher's Note: MDPI stays neutral with regard to jurisdictional claims in published maps and institutional affiliations.

(C) 2020 by the authors. Licensee MDPI, Basel, Switzerland. This article is an open access article distributed under the terms and conditions of the Creative Commons Attribution (CC BY) license (http:/ / creativecommons.org/licenses/by/4.0/). 